\title{
The heat capacity of the restricted primitive model electrolyte
}

\author{
Erik Luijten* and Michael E. Fisher \\ Institute for Physical Science and Technology, University of Maryland, College Park, MD 20742 \\ Athanassios Z. Panagiotopoulos \\ Department of Chemical Engineering, Princeton University, Princeton, NJ 08540
}

(November 2, 2000)

\begin{abstract}
The constant-volume heat capacity, $C_{V}(T, \rho)$, of the restricted primitive model (RPM) electrolyte is considered in the vicinity of its critical point. It is demonstrated that, despite claims, recent simulations for finite systems do not convincingly indicate the absence of a divergence in $C_{V}(T, \rho)-$ which would point to non-Ising-type criticality. The strong qualitative difference between $C_{V}$ for the RPM and for a Lennard-Jones fluid is shown to result from the low critical density of the former. If one considers the theoretically preferable configurational heat-capacity density, $C_{V} / V$, the finite-size results for the two systems display qualitatively similar behavior on near-critical isotherms.
\end{abstract}

The critical behavior of Coulombic systems continues to be subject to debate. Whereas it is generally accepted that the critical behavior of the gas-liquid transition in simple liquids belongs to the three-dimensional (3D) Ising universality class, the situation in ionic solutions is considerably more obscure. At sufficiently low temperatures, these solutions exhibit separation into two phases with different density, driven primarily by the Coulombic forces between the charged constituents. Experimentally, both classical (as one might guess from the longrange character of the ionic forces) and Ising-type critical behavior (as might be explained by the effects of Debye screening) have been reported: see, e.g., Refs. 11.2. Other possible scenarios entail a crossover from classical to Ising-type behavior at considerably smaller reduced temperatures than in simple fluids or even the existence of a different type of criticality. 1 , 6

In view of the significance of electrolytes and ionic systems in many domains, a clear understanding of their critical behavior is of interest. It is, therefore, disconcerting that even for the simplest model thought to capture the salient features of such systems, namely, the restricted primitive model (RPM), the universality class has not yet been established beyond reasonable doubt. The RPM consists of a mixture of hard spheres of uniform diameter $\sigma$, half of which carry a charge $+q$ and half a charge $-q$. Its critical behavior has been analyzed by both analytical and numerical means. Analytically, a fairly satisfactory description of the critical region (except for the nature of the criticality) has been obtained from Debye-Hückel theory supplemented by Bjerrum's concept of ion pairing and allowance for the solvation of dipolar-ion pairs in the ionic fluid. 3 However, lack of a sufficiently adequate formulation at the mean-field level has hindered the development of a renormalization-group treatment, see, e.g., Ref. \&. Furthermore, simulations have also encountered serious difficulties, not only be- cause of the long-range nature of the interactions, but, in particular, because of the low value of the critical temperature and the resulting presence of many strongly bound ion pairs. 8 The limited statistical accuracy and range in system sizes that have been reliably accessed have hampered detailed numerical analysis.

This note has been inspired by recent work by Valleau and Torrie (VT), 9 who performed numerical simulations of the RPM using a temperature-and-depsityscaling Monte Carlo method. Other simulations 5010 focused mainly on the coexistence curve below $T_{c}$. Experimentally, observations of the coexistence curve as $T \rightarrow T_{c}-$ have been revealing of universality class (with $\beta_{\text {Ising }} \simeq 0.326$ and $\beta_{\text {classical }}=\frac{1}{2}$ ) or of crossover behavior. In simulations, however, finite-size effects preclude the estimation of the coexistence curve close to $T_{c}$ : Wilding and Bruce 11 have devised a finite-size scaling technique for analyzing the corresponding Monte Carlo data which has led to fairly precise and seemingly rather reliable estimates of the critical temperature, $T_{c}$, and to reasonable estimates of the overall ionic critical density, $\rho_{c}$. However, their technique presupposes Ising-type criticality and has not, therefore, provided any effective criteria for ruling out (or, possibly, revealing) other types of criticality.

By contrast, $\mathrm{VT}^{9}$ focused on the heat capacity at constant volume, $C_{V}(T, \rho)$, in the one-phase region both as a function of density, $\rho$, near $T_{c}$ and on approach to criticality from above. In a classical, or van der Waalstype system $C_{V}$ remains finite as $T \rightarrow T_{c}+$ on the critical isochore, $\rho=\rho_{c}$, whereas in an Ising-type system, $C_{V}\left(T, \rho_{c}\right)$ diverges to infinity, albeit weakly with an exponent $\alpha \simeq 0.109$. As one passes through $T_{c}$ from above in a classical system, $C_{V}\left(T, \rho_{c}\right)$ undergoes a positive jump discontinuity and decreases smoothly thereafter: see Fig. 1; an Ising-type fluid exhibits a $\left|T-T_{c}\right|^{-\alpha}$ singularity falling rapidly from infinity as $T$ decreases.

*Electronic address: luijten@ipst.umd.edu 
Accordingly, VT argued that an examination of $C_{V}(T, \rho)$ for the RPM for $T \gtrsim T_{c}$ and, in particular, comparison with simulations of a Lennard-Jones (LJ) model fluid (for which Ising-type or close-to-Ising-type behavior may be accepted) should provide an effective diagnostic of critical behavior. On the basis of the simulations they undertook and presented, VT concluded that little if any evidence of a rise in specific heat was present in the RPM. This suggested that criticality in the RPM might be classical in nature or, at least, characterized by crossover rather close to $T_{c}$.

While we acknowledge the potential value of the VT approach, we find, as will be explained, that we cannot accept the validity of their analyses or of the conclusions they draw. Indeed, although Ising and classical behavior are essentially different in the thermodynamic limit, they are far more difficult to distinguish in the small systems that are accessible to numerical simulations. Specifically, VT studied the heat capacity (i) along the estimated critical isochore (for $T>T_{c}$ ) and (ii) along the anticipated critical isotherm, for a wide range of densities. In the first case, as mentioned, they found no signs of a divergence in $C_{V}\left(T, \rho_{c}\right)$. In the second case, no (finite-size rounded) peak was seen near the critical density. It is this latter observation that VT advance as strong evidence against Ising-type critical behavior in the restricted primitive model, since, as they illustrated, $C_{V}\left(T_{c}, \rho\right)$ in a LennardJones fluid exhibits a clear, system-size-dependent peak in the vicinity of $\rho=\rho_{c}$. Here, we reconsider this evidence, which stands unchallenged to date, either through new simulations or via a reanalysis of the VT data.

Consider, first, the heat capacity along the critical isochore. VT observe that $C_{V}\left(T, \rho_{c}\right)$ increases almost linearly upon approach to $T_{c}$ from above, with no evidence of a divergence. They do, however, remark that this might be due to the fact that all their observed temperatures lay within the regime of finite-size rounding, where the correlation length is restricted by the system size. We feel, rather, that it is the constraint $t \equiv\left(T-T_{c}\right) / T_{c}>0$ that might lead to premature conclusions. In Fig. 1 we show the specific heat at constant volume for an infiniterange, van der Waals or mean-field lattice gas (in which all particles interact equally) for a number of system sizes 12. 13 The plots for density $\rho=\rho_{c} \equiv \frac{1}{2} \rho_{\max }$ represent the behavior along the critical isochore, whereas the curves for $\rho=\frac{3}{4} \rho_{c}$ and $\rho=\frac{5}{4} \rho_{c}$ (the system being symmetric around $\rho_{c}$ ) illustrate the behavior along a noncritical isochore. As expected, the peak heights are lower if $\rho \neq \rho_{c}$; but the qualitative behavior of the specific heat evidently persists even for relatively large deviations from the critical isochore. (Thus, even moderately large errors in the estimate of $\rho_{c}$ for the RPM should not affect qualitative conclusions.)

The crucial point, however, is that (despite the absence of a divergence of $C_{V}\left(T, \rho_{c}\right)$ in the thermodynamic limit) the mean-field plots display pronounced size-dependent maxima for $T<T_{c}$. Indeed, even though these peak heights must saturate, 13 whereas they diverge for an
Ising-type system with short-range interactions, the behavior of small systems is qualitatively very similar in both cases. In particular, the specific heats of finite 3D Ising models and hard-core square-well fluids display maxima below $T_{c} 14$ Thus, it may be difficult to distinguish the two types of behavior unless one has a sufficiently large range of system sizes to allow extrapolation of the peak height and position. Certainly, the linear increase of $C_{V}(T, \rho)$ for $T \rightarrow T_{c}+$ for a given system size, as VT observed for the RPM with $\rho \simeq \rho_{c}$, would seem to convey little information regarding the nature of the critical behavior. This is basically a consequence of the fact that for finite 3D systems the specific-heat maxima seem invariably to occur below the true, limiting critical temperature.

In order to illustrate this point more concretely, we have carried out high-resolution Monte Carlo simulations of a discretized version of the RPM. 15 This model differs from the continuum RPM only in that the positions of the ions are restricted to lattice sites: the degree of discretization is determined by the ratio, $\zeta$, of the ion diameter $\sigma$ to the lattice spacing $a$. The continuum limit is recovered by taking $\zeta \rightarrow \infty$. It has been shown 15 that already for the small discretization parameters $\zeta=3$, 4 , and 5 , this model exhibits a liquid-vapor transition like the continuum RPM, with a coexistence curve that approaches that of the continuum model very closely. We have focused on $\zeta=5$, and carried out histogram-reweighted grandcanonical simulations for simple cubic lattices of sizes up to $L=10 \sigma$, which corresponds to $(\zeta L / \sigma)^{3}=125000$ possible ion positions. Periodic boundary conditions were employed.

The strong ion pairing at low temperatures makes grand-canonical simulations especially time consuming. However, we view canonical simulations as inherently dangerous owing to the important role of density fluctuations in the vicinity of the critical point. (See also further comments below.) A detailed study of these and related data is in progress 16 but preliminary examination suggests a reduced critical temperature $T_{c}^{*} \simeq 0.051$ and a critical density $\rho_{c}^{*} \simeq 0.068$. (See, e.g., Refs. 116 for the standard definitions of reduced units for the RPM.) Our estimates agree with the suggestion of Ref. 15 that the RPM with $\zeta=5$ has a $T_{c}^{*}$ slightly higher than that of the continuum RPM $\left(T_{c}^{*} \simeq 0.049\right.$ (6) 10$)$, although the estimate of $\mathrm{VT}$ is $T_{c}^{*} \simeq 0.052$. Figure 2 shows the specific heat of this model for $\rho \simeq \rho_{c}$ over a relatively wide temperature range around $T_{c}$. As in Fig. 1, system-size dependent maxima are indeed observed at temperatures that clearly approach $T_{c}$ from below. Their variation with $L$ suggests quite strongly that Ising-type behavior cannot be ruled out.

On the other hand, VT's results for the density dependence of $C_{V}$ at $T=T_{c}$ present, at first sight, a more challenging puzzle. Indeed, their data appeared to reveal a remarkable difference between the RPM and a Lennard-Jones fluid. For the former, $C_{V} / N k_{\mathrm{B}}$ decreased monotonically with increasing density, without any ev- 
ident marked dependence on system size. The corresponding curves VT present for a Lennard-Jones fluid, on the contrary, exhibit a pronounced peak in the vicinity of the critical density $\rho_{c}$ that, furthermore, increases with system size.

Why is a peak for the RPM apparently absent? Without doubt, any maximum or divergence related to criticality in the RPM will be rounded and shifted in finite systems, irrespective of the actual universality class. Furthermore, one must be prepared for strong finite-size effects that are likely to be distorted relative to LJ-type model fluids in light of the shape of the RPM coexistence curve, which is highly asymmetric. 1 B This expectation is indeed confirmed by Fig. 3, where the continuous plots show our simulation data for the specific heat of the discretized RPM for six different system sizes at $T^{*}=0.051 \simeq T_{c}^{*}$. For each system size there is a clear maximum, at a density that appears to approach the critical density from below. Unfortunately, however, the data of VT (shown for their system size $N=192$ ) did not extend to sufficiently low densities to cover these peaks. Also noteworthy is that their data actually agree rather well with ours for the discretized RPM, at a system size between $L=5 \sigma$ and $L=6 \sigma$, even though this is considerably smaller than the dimension $\tilde{L}_{N} \equiv(N / \rho)^{1 / 3} \simeq 13 \sigma$ suggested by the particle number $N=192$. This difference might be due to the $\zeta \overline{\overline{ }} 5$ discretization; but if $T_{c}$ really is lower for the RPM, $\mathrm{E}$ VT's data were taken at an isotherm somewhat above $T_{c}$. On the other hand, one cannot exclude the possibility that the difference reflects a relative limitation of the canonical simulations performed by VT. The fixed particle number may, in effect, suppress the characteristic density fluctuations, with a consequential relative suppression and enhanced rounding of various maxima in finite systems. This might also explain why VT observe negligible changes in $C_{V}$ when increasing the number of ions by 50\% from 128 to 192 . However, the precise nature of the finite-size effects themselves is currently unclear in the framework of the $T$-and$\rho$-scaling Monte Carlo used by VT.

Now consider the qualitative differences observed by VT between the specific heats of the RPM and the LJ fluid. These differences also prove to be a consequence of the low critical density of the RPM, compared to $\rho_{c}$ for simple fluids; they do not reflect any significant possible difference in the nature of the critical behavior of the two systems. To see this, recall that in comparing the configurational heat capacity at constant volume for different fluids, or for a fluid and a lattice model, the more basic quantity for criticality and phase separation is the heat capacity per unit volume (or $C_{V}$ density) rather than the specific heat (or heat capacity per particle).17 In addition to the arguments presented in Ref. 17, namely the greater naturalness of the grand-canonical ensemble and the expectation that spatial fluctuations most directly characterize critical divergences, note that the field-theoretic viewpoint of critical phenomena and the renormalizationgroup approach 18 bear out the conclusion that the num- ber of degrees of freedom per unit volume plays the most fundamental role.

Thus, in Fig. 1 we have plotted the heat-capacity density, $C_{V} / V k_{\mathrm{B}}$, for the RPM as a function of $\rho$ for $T^{*}=0.051$, with a "harmless background" proportional to $\rho$ subtracted. Instead of a monotonically decreasing function, we now obtain a net "energy fluctuation" that displays a clear maximum as a function of $\rho$, of a height that increases systematically with size. If we replot VT's data for the Lennard-Jones fluid in the analogous way, as in Fig. 5 , we find similar behavior: indeed, the subtracted heat-capacity density of the LJ fluid on the critical isotherm exhibits clear finite-size maxima as a function of the density, with a systematic size dependence that, as for the RPM, suggests a monotonic increase (as $L$ or $N \rightarrow \infty$ ) peaking in the vicinity of the critical density.

In summary, the constant-volume heat capacity is a useful quantity in the study of the critical behavior of the restricted primitive model for ionic fluids. However, as we have demonstrated, care must be exercised before concluding that a maximum or a critical divergence is absent. Along both the critical isochore and the critical isotherm, numerical results for finite systems exhibit clear maxima, contrary to the suggestions of Ref. 9. Furthermore, inasfar as one observes an overall qualitative difference between the specific heat for the RPM and a fluid with Lennard-Jones interactions, the effect is primarily due to the large difference in critical densities. It has no relevance to possible differences in critical behavior - for which our current data allow few definitive statements. Conversely, if one considers the heat-capacity density, the two systems exhibit qualitatively rather similar behavior. Whether, ultimately, that reflects the same or distinct critical universality classes remains to be determined on the basis of fluctuations not only of the energy, as observed in the specific heat, but also of fluctuations in density. 14.16

\section{ACKNOWLEDGMENTS}

We thank Dr. G. Orkoulas for informative discussions. The support of the National Science Foundation (through Grant No. CHE 99-81772 to M.E.F.) and of the Department of Energy, Office of Basic Energy Sciences (through Grant No. DE-FG02-98ER14858 to A.Z.P.) is gratefully acknowledged.

\footnotetext{
${ }^{1}$ M. E. Fisher, J. Stat. Phys. 75, 1 (1994).

2 M. E. Fisher, J. Phys.: Condens. Matter 8, 9103 (1996) and references therein.
} 
${ }^{3}$ M. E. Fisher and Y. Levin, Phys. Rev. Lett. 71, 3826 (1993); Y. Levin and M. E. Fisher, Physica A 225, 164 (1996).

${ }^{4}$ A. G. Moreira, M. M. Telo da Gama, and M. E. Fisher, J. Chem. Phys. 110, 10058 (1999) and references therein.

${ }^{5}$ J. M. Caillol, J. Chem. Phys. 100, 2161 (1994).

${ }^{6}$ G. Orkoulas and A. Z. Panagiotopoulos, J. Chem. Phys. 101, 1452 (1994); 110, 1581 (1999).

${ }^{7}$ J. C. Shelley and G. N. Patey, J. Chem. Phys. 103, 8299 (1993).

8 J. M. Caillol and D. Levesque and J. J. Weis, Phys. Rev. Lett. 77, 4039 (1996).

${ }^{9}$ J. Valleau and G. Torrie, J. Chem. Phys. 108, 5169 (1998).

${ }^{10}$ Q. Yan and J. J. de Pablo, J. Chem. Phys. 110, 9509 (1999).

${ }^{11}$ N. B. Wilding and A. D. Bruce, J. Phys.: Condens. Matter 4, 3087 (1992).

${ }^{12}$ R. J. Baxter, Exactly Solved Models in Statistical Mechanics (Academic, London, 1982).

${ }^{13}$ E. Luijten, Interaction Range, Universality, and the Upper Critical Dimension (Delft University Press, 1997).

${ }^{14}$ G. Orkoulas, A. Z. Panagiotopoulos, and M. E. Fisher, Phys. Rev. E 61, 5930 (2000).

15 A. Z. Panagiotopoulos and S. K. Kumar, Phys. Rev. Lett. 83, 2981 (1999).

${ }^{16}$ E. Luijten, M. E. Fisher, and A. Z. Panagiotopoulos, in preparation.

17 M. E. Fisher, Phys. Rev. 136, A1599 (1964).

18 See, e.g., M. E. Fisher, Rev. Mod. Phys. 46, 597 (1974); 70, 653 (1998).

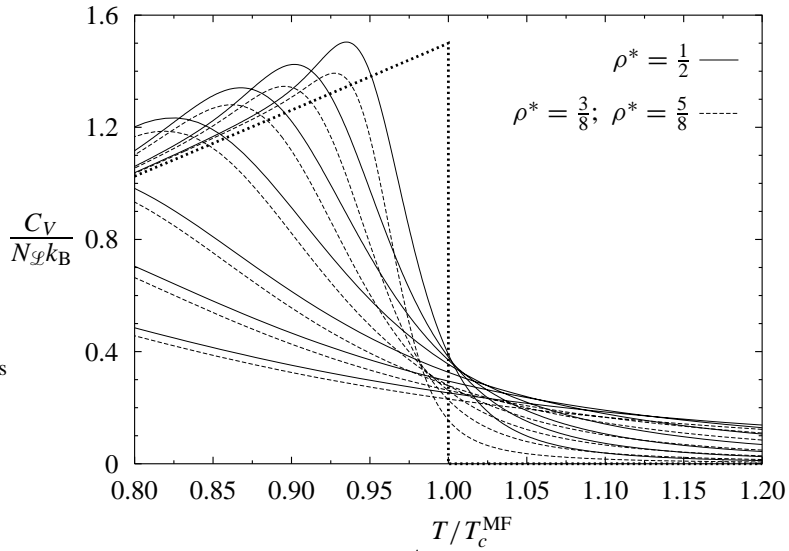

FIG. 1. The specific heat, $C_{V} / N_{\mathcal{L}} k_{\mathrm{B}}$, for a grand-canonical lattice gas with identical interactions between all particle pairs, near the mean-field critical temperature $T_{c}^{\mathrm{MF}}$. The solid curves derive (in order of increasing peak height) from finite systems of $N_{\mathcal{L}}=10,20,40,100,200,400,1000$ lattice sites, for a mean particle density $\rho=\rho_{c}=\frac{1}{2} \rho_{\max }$. The corresponding dashed curves pertain to densities $\rho=\frac{3}{4} \rho_{c}$ and $\rho=\frac{5}{4} \rho_{c}$. The bold dotted curve represents the thermodynamic limit, $N_{\mathcal{L}} \rightarrow \infty$, for $\rho=\rho_{c}$. The maxima of the finite-size curves saturate at approximately 1.657, rather than at the thermodynamic maximum $\frac{3}{2}$. 13

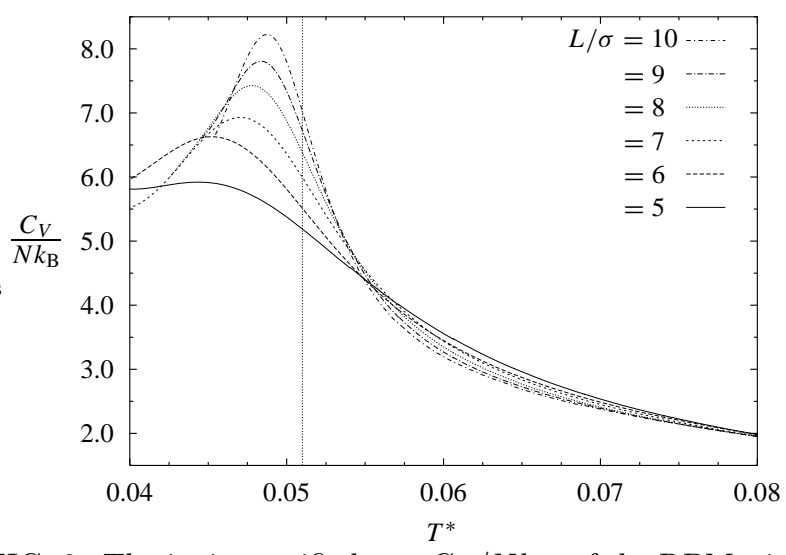

FIG. 2. The ionic specific heat, $C_{V} / N k_{\mathrm{B}}$, of the RPM with discretization parameter $\zeta=5$, along the estimated critical isochore. Clear peaks rounded by finite size are evident below the estimated critical temperature (vertical dashed line), although the behavior above $T_{c}$ conveys significantly less information.

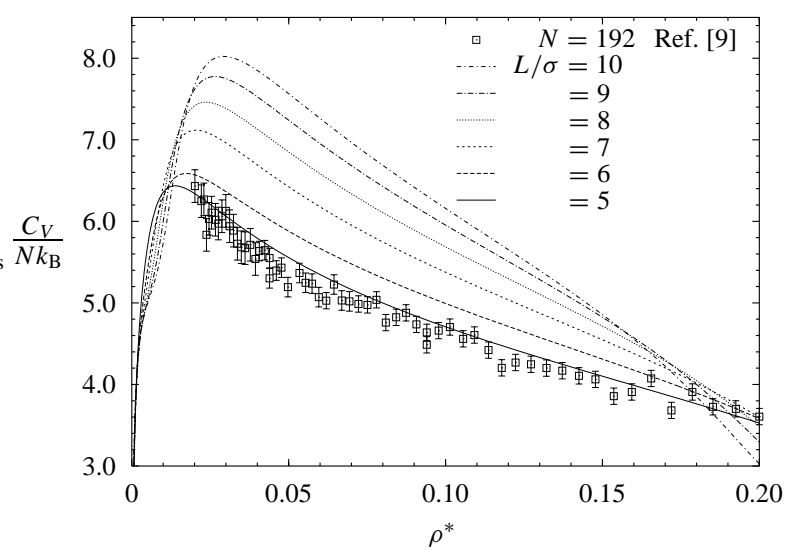

FIG. 3. The constant-volume specific heat on the estimated critical isotherm of the discretized RPM (for $\zeta=5$ ), compared with the corresponding VT data (Ref. 9).

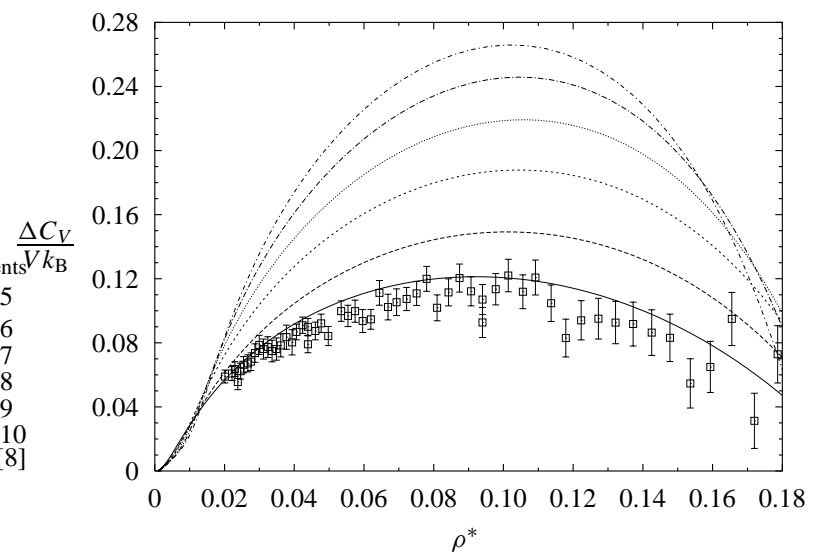

FIG. 4. As in Fig. 3, but now the heat-capacity density is plotted and a linear "background term" $C_{V}^{0} / V k_{\mathrm{B}}=3.5 \rho^{*}$ has been subtracted. 


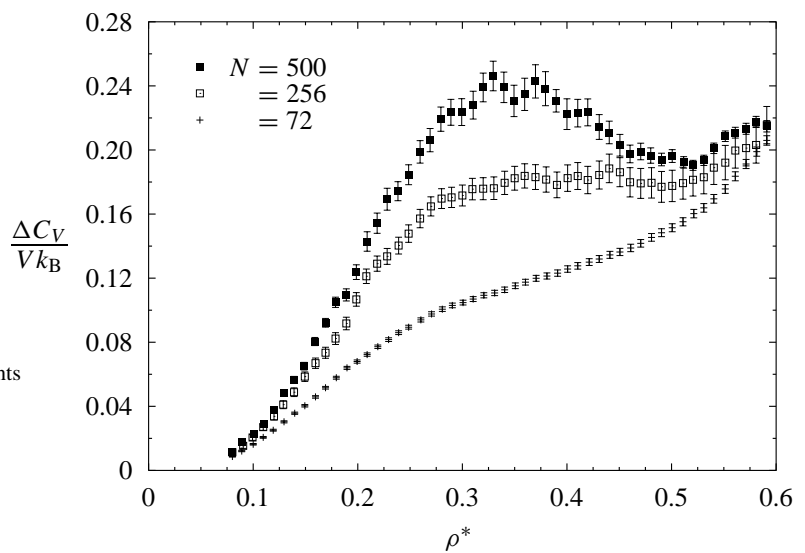

FIG. 5. The heat-capacity density for a Lennard-Jones fluid, as derived from the data of Valleau and Torrie (Ref. 9). As in Fig. 1, a "background" $C_{V}^{0} / V k_{\mathrm{B}}=1.6 \rho^{*}$ has been subtracted from $C_{V} / V k_{\mathrm{B}}$. 